\section{GENE EXPRESSION}

\section{Recruiting modifiers}

Nat. Biotechnol. https://doi.org/10.1038/s41587019-0296-7 (2019)

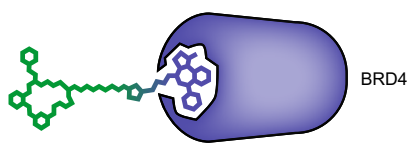

Credit: Nature

Recent work has utilized programmable DNA-binding ligands or catalytically inactive Cas9 to deliver modulators of chromatin modifications to a particular gene locus to alter gene expression. However, these approaches are unable to tune gene expression in a dose-dependent manner using endogenous enzymes. To address this limitation, Chiarella et al. devised a system that utilizes a catalytically inactive Cas9 linked to a FK506-binding protein (FKBP) and a chemical epigenetic modifier (CEM). The CEM consists of FK506 linked to a molecule that binds and recruits chromatin-modifying enzymes, such as the BRD family of bromodomains and CBP, to a locus defined by the guide RNA. The titration of CEMs resulted in a dosedependent activation of gene expression 48 $\mathrm{h}$ after treatment, boosting RNA transcripts from weakly expressed genes with a range of 10-20 fold. RNA-seq analysis confirmed that CEM treatment resulted strong BRD4 recruitment to the promoter region targeted by guide RNA. Overall, the CEM system offers a precise means of stimulating gene expression in a dose-dependent fashion.

$G M$

https://doi.org/10.1038/s41589-019-0440-1

\section{TARGETED PROTEIN DEGRADATION}

\section{Trashing transcription}

Cancer Cell 36, 498-511 (2019)

Although transcription factors are thought to be undruggable owing to lack of active sites, several inhibitors for transcription factor STAT3 have been developed by targeting its $\mathrm{SH} 2$ domain to disrupt its dimerization. However, it remains challenging to develop selective STAT3 inhibitors for therapeutic purpose because of the high homology of SH2 domains among STAT family members and the residual activity of STAT3 monomers. Bai et al. utilized proteolysis-targeting chimera (PROTAC) technology to achieve a highly selective STAT3 degrader designated as SD-36 using an optimized STAT3 inhibitor with moderate selectivity linked to the cereblon ligand lenalidomide. SD-36 reduced the protein levels of total and active STAT3 in a time- and dose-dependent manner, while RNA-seq and proteomics analysis showed that SD-36 exhibited high selectivity toward STAT3. Furthermore, SD-36 is $>1,000$ times more potent than its corresponding STAT3 inhibitor SI-109 in inhibiting the transcriptional activity of
Depending on genetic susceptibility and environmental conditions, the inflammatory heart disease myocarditis can progress into lethal cardiomyopathy, and this transition is associated with autoimmune responses against myosin heavy chain $6(\mathrm{MYH6})$. To examine the role of the microbiota as an environmental component driving lethal disease, Gil-Cruz et al. generated mice with myocarditis due to expression of a MYH6specific T cell receptor (TCRM). The mice only developed lethal disease associated with heart-infiltrating TCRM cells if a microbiota was present; germ-free mice did not develop cardiomyopathy. In silico searching revealed Bacteroides thetaiotaomicron $(B$. theta) and Bacteroides faecis $\beta$-galactosidase ( $\beta$ gal) peptides with high similarity to $M Y H 6$, suggesting that $B$. theta and $B$. faecis $\beta$ gal may contribute to myocarditis. The $B$. theta $\beta$ gal could activate TCRM T cells, whereas its deletion blocked accumulation of immune cells in the myocardium of TCRM mice. Similarly, antibiotic treatment prevented lethal cardiomyopathy and reduced cardiac inflammation. The authors found that patients with human acute myocarditis had improved symptoms when their $B$. theta immunoreactivity was reduced. These results suggest that inflammatory cardiomyopathy can be driven by bacterial peptide mimics derived from the intestinal microbiota that activate heart-specific T cells.
STAT3. SD-36 induced growth inhibition in a subset of cancer cell lines with activated STAT3 and achieved complete tumor regression in several xenograft models in mice. This study underlines the advantage of PROTAC technology in pharmaceutical development and provides a successful example of targeting transcription factors.

https://doi.org/10.1038/s41589-019-0442-z

\section{BIOORTHOGONAL CHEMISTRY}

\section{Forging a new connection}

J. Am. Chem. Soc. 141, 18644-18648 (2019)

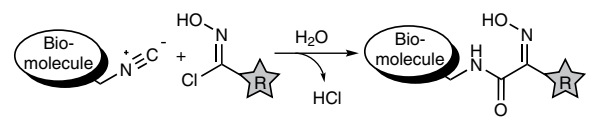

Credit: ACS

Bioorthogonal chemical reactions provide facile routes to generate modified biological molecules. Useful bioorthogonal reactions must be both efficient and chemoselective, utilize moieties rarely (if at all) found in natural biomolecules, and generate stable ligation products. In the interest of developing new reactions that generate minimal ligation moieties, Schäfer and Monaco et al. identified the bioorthogonal capacity for the reaction of isonitriles and chlorooximes to generate hydroxyimino amides. The authors demonstrated that this reaction is chemoselective, is robust at physiological $\mathrm{pH}$ and temperature, and accommodates a variety of appended moieties. Not only is the reaction compatible with aqueous conditions (unlike many organic reactions), but water is actually a necessary reagent both to activate the chlorooxime and to resolve the final intermediate. In addition, the reaction is compatible with living cells, as demonstrated by metabolic labeling of cell-surface glycans, and orthogonal to the commonly used strain-promoted azide-alkyne cycloaddition reaction, enabling dual labeling. With its selectivity, versatility, and small size, the isonitrile-chlorooxime ligation is a robust new addition to the bioorthogonal reaction toolbox. 